\title{
Sensorineural hearing loss in adult onset Still's diseas
}

\author{
HARRY M MARKUSSE, BEREND STOLK, ANDEL G L VAN DER MEY, \\ JOHANNA M DE JONGE-BOK, AND KAREL J HEERING
}

From the Departments of Rheumatology, Medicine, and Otorhinolaryngology, University Hospital, Leider? and the Departments of Rheumatology and Medicine, Bleuland Hospital, Gouda

SUMMARY A 26 year old man with a nine year history of adult onset Still's disease (AOSD developed sensorineural hearing loss during an exacerbation of his disease. This complication has not previously been described in association with adult onset Still's disease. He respondee favourably to steroid treatment.

Key words: deafness, juvenile arthritis.

A patient with a polycyclic systemic adult onset Still's disease (AOSD) is reported, who developed a sensorineural hearing loss during an exacerbation of his disease. The hearing loss and the systemic activity of AOSD were both controlled by corticosteroid treatment. To the best of our knowledge the association of sensorineural hearing loss with AOSD has not been published before.

\section{Case report}

In 1987 a 26 year old man presented with a nine year history of periodic illness which, in 1978, had been evaluated for quotidian spiking fever, evanescent rash, arthralgias, lymphadenopathy, leucocytosis, and liver enzyme abnormalities. At the time negative results were obtained in the following investigations: cultures of blood, stools and urine, RoseWaaler test, antinuclear antibody, muscle biopsy, bone marrow biopsy, and $x$ ray films of the chest, gall bladder, stomach, and sacroiliac joints. A diagnosis of AOSD was made, and salicylate treatment resulted in symptomatic improvement. During the following years splenomegaly was found and several exacerbations were successfully treated with salicylates. A flare up in 1986 responded to indomethacin. Another exacerbation in February 1987, however, failed to improve with indomethacin

Accepted for publication 24 December 1987.

Correspondence to Dr Harry M Markusse, Staffcentre Rheumatology Building 1, C2-Q, University Hospital, PO Box 9600, 2300 RC Leiden, The Netherlands.
$150 \mathrm{mg}$ daily. On 20 March 1987 he was admitted to hospital still suffering from quotidian spiking fevers and arthritis of the ankles and metacarpophalange joints, an evanescent rash of the forearms ante hands, and weight loss of $6 \mathrm{~kg}$. A few days afte? admission he gradually developed hearing loss and tinnitus. Indomethacin treatment was stopped on $2 \overline{3}$ March 1987, but despite this a pure tone audiogram taken two days later showed a sensorineural hearing loss.

Investigations showed erythrocyte sedimentation rate (ESR) $72-94 \mathrm{~mm} / 1 \mathrm{st} \mathrm{h}$, white cell count (WCG) $9 \cdot 5-17.2 \times 10^{9} / 1$, with a normal differential, and slightly raised liver enzymes. Creatinine, calcium glucose, thyroxine, serum protein immunoele 6 trophoresis, vitamins $B_{1}, B_{6}$, and $B_{12}$, and serum folate, and 24 hour urinary calcium excretion we normal.

The following investigations failed to show aros abnormality: rheumatoid factor titre, antinucleas antibodies, antibodies to native DNA, angiotensio converting enzyme, cryoglobulins, hepatitis B antiّ gen, and syphilis serology. A lumbar puncture yielded clear, colourless cerebrospinal fluid with lo cell counts and no protein; cultures of blood, stool ${ }_{2}$ urine, and cerebrospinal fluid showed no growth There was no sequential serological evidence for recent infection with chlamydia, varicella zost virus, herpes simplex virus, echovirus, Cocksack virus $\mathrm{A}$ and $\mathrm{B}$, cytomegalovirus, adenovirus, mum $P^{\circ}$ virus, or Epstein-Barr virus. $x$ Rays of the chest an hands, electrocardiography, slit lamp examinatiog 

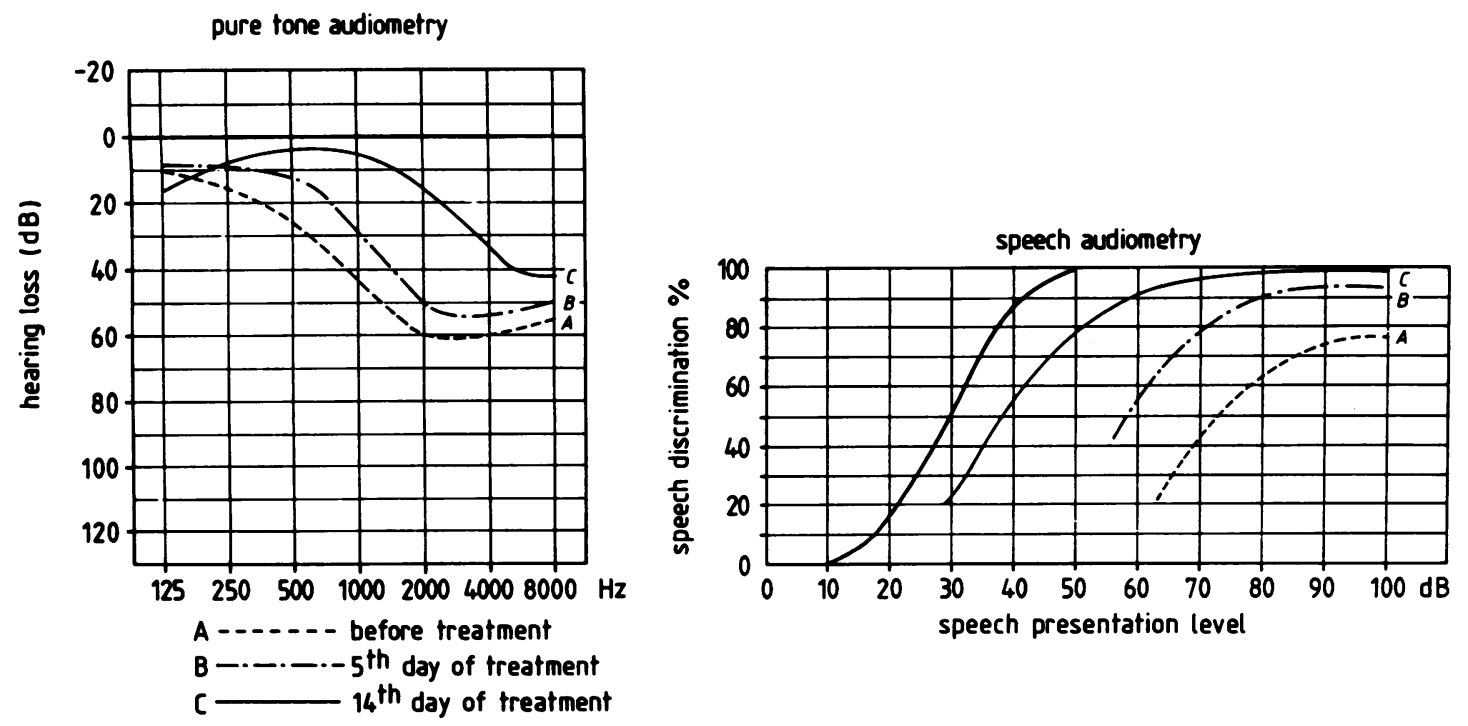

Fig. 1 Pure tone and speech audiograms. Because both ears disclosed the same abnormalities only the audiograms of the right ear are shown.

of the eyes, and pulmonary function tests were normal. Echography of the abdomen showed splenomegaly. Biopsy specimens from the nasal mucosa and the rectal mucosa and needle aspiration of the abdominal subcutaneous fat showed no abnormalities. A computed tomographic scan of the skull base and brain were normal. Audiological examination, including brainstem evoked response audiometry (BERA), showed a (cochlear type) sensorineural hearing loss of 40-50 dB in the speech frequencies, without any evidence of middle ear or retrocochlear localisation of the lesion (Fig. 1A).

The audiometric findings made two days and 19 days after refraining from indomethacin treatment were essentially identical (Fig. 1A). Twenty one days after the withdrawal of indomethacin, prednisone $60 \mathrm{mg}$ daily was started. A prompt response was manifested by a fall in temperature, resolution of the arthritis, and a subjective improvement in the patient's hearing.

The fifth day after starting prednisone treatment there was clear improvement in both pure tone and speech audiometry (Fig. 1B). On the 14th day of treatment the improvement for both ears was even more obvious (Fig. 1C).

After four weeks the prednisone dosage was tapered to $25 \mathrm{mg}$ daily; the ESR was $10 \mathrm{~mm} / \mathrm{h}$, WCC $7 \cdot 5 \times 10^{9} / 1$, and the liver enzymes were normal. There was no longer any fever, rash, or arthritis, the tinnitus gradually disappeared, and further progress in hearing acuity in both ears was found on audiometric control.

\section{Discussion}

Adult onset Still's disease is a cyclic systemic inflammatory disorder mostly diagnosed before the age of 35 , characterised by high spiking quotidian fever, arthritis, and evanescent rash. Leucocytosis, lymphadenopathy, serositis, hepatosplenomegaly, and hepatic enzyme abnormalities are other features of this disease. ${ }^{1-3}$ In patients with a systemic disease and hearing loss, conditions such as Cogan's syndrome, polyarteritis nodosa, Wegener's granulomatosis, relapsing polychondritis, and systemic lupus erythematosus have to be considered. ${ }^{4}$ In this case these diseases were conclusively excluded by the long history, the clinical features, and the comprehensive investigations. This patient fulfilled all the AOSD criteria proposed by Calabro and Londino. ${ }^{6}$ Although the predictive value of these criteria has never been tested, we believe that in this case a diagnosis of AOSD is justified. The cyclic systemic disease activity with only mild articular involvement was characteristic. After nine years he developed hearing loss and tinnitus during another exacerbation manifested by generalised malaise, weight loss, high spiking fever, mild arthritis, leucocytosis, raised hepatic enzyme concentrations, splenomegaly, and an evanescent rash.

Tinnitus, normal otoscopy, pure tone audiometry, speech audiometry, tympanometry, and BERA pointed to cochlear involvement.

Many drugs may cause audiovestibular toxicity, but we could not find any report of indomethacin 
related sensorineural hearing loss. The time between the introduction of indomethacin treatment and the onset of cochlear symptoms, the lack of improvement of the hearing after indomethacin was stopped, and the striking amelioration of the cochlear function after starting prednisone treatment virtually exclude indomethacin as a cause of this hearing loss.

Extensive viral and bacteriological studies were unremarkable and a normal computed tomogram of the skull and a normal lumbar puncture made a central nervous system process unlikely.

The simultaneous improvement of this patient's cochlear function and his general condition immediately after the start of prednisone treatment make AOSD the most likely cause of the hearing loss.

\section{References}

1 Larson E B. Adult Still's disease. Evolution of a clinica syndrome and diagnosis, treatment and follow-up of 17 patients. Medicine (Baltimore) 1984; 63: 82-91.

2 Cush J J, Medsger Jr T A, Christy W C, Herbert D Cooperstein L A. Adult-onset Still's disease. Arthritis Rheur 1987; 30: 186-94.

3 Wouters J M G W, van de Putte L B A, Cooperating Centresn Adult-onset Still's disease; clinical and laboratory features, treatment and follow-up of 45 cases. Adult-onset Still's disease $\overrightarrow{\vec{D}}$ Nijmegen, 1986: 19-39. (Thesis.)

4 Haynes B F, Kaiser-Kupfer M I, Mason P, Fauci A S. Cogaț syndrome. Medicine (Baltimore) 1980; 59: 426-41.

5 Cardarelli D D, Rejowski J E, Corey J P. Sensorineural hearing loss in lupus erythematosus. Am J Otolaryngol 1986; 7: 210-3

6 Calabro J J, Londino Jr A V. Adult onset Still's disease. Х Rheumatol 1986; 13: 827-8. 